\title{
Low-temperature resistance fluctuation in disordered conductors
}

\author{
N KUMAR \\ Department of Physics, Indian Institute of Science, Bangalore 560012, India
}

\begin{abstract}
At low temperatures the electron elastic mean free path in a disordered conductor can become much smaller than the inelastic mean free path (or more precisely the Thouless length) which in turn may be comparable with, or even larger than the sample size. In this quantum regime, the electrical resistance is dominated by the coherence effects that eventually lead to the now well-known weak or strong localization. Yet another remarkable manifestation of the quantum coherence is that it makes the resistance non-additive in series and, more importantly, non-self averaging, thus replacing the classical Ohm's law with a quantum Ohm's law describing statistical fluctuations. In this paper, we report on some of our recent work on the statistics of these "Sinai" fluctuations of residual resistance for one and higher space dimensions $(d)$. In particular we show that the physics at the mobility edge may be dominated by these fluctuations. We also show that an external electric field tends to harness these fiuctuations. Some observational consequences such as $1 / f$-noise at low temperatures are discussed. Our approach is based on invariant imbedding extended by us for this purpose.
\end{abstract}

Keywords. Disordered conductors; low-temperature resistance fluctuation; residual resistance; mobility edge; Sinai fluctuations.

PACS No. $72 \cdot 10$

\section{Introduction}

Theoretical physics of disordered systems frequently involves a convenient artifice of averaging of physical quantities of interest over the probability distribution of the underlying quenched randomness which is assumed to be given. This is despite the fact that the experiment is performed on a given sample. The rationale for this mathematical artifice is that for a macroscopic sample, i.e., in the thermodynamic limit, different parts of the sample may be taken as different instances of the sample. Thus, if the quantity of interest is extensive i.e., expressible as an integral of a local density over the sample volume, then the observed value is reproduced by the ensemble averaging which then becomes a matter of convenience. There are, however, quantities which are not self-averaging in the above sense. Well-known examples are from the statistical mechanics of disordered systems, e.g., the archetypal spin glass. Here the partition function and several susceptibilities are in fact not self-averaging, but the free energy is. In the following we will be concerned with the (residual) resistance of a disordered conductor at low temperatures which is dominated by quantum coherence effects (Kumar 1985; Kumar and Jayannavar 1986; Kumar and Mello 1985). The latter will be shown to make the resistance non-additive and non-self averaging. This was already noted for the case of one-dimensional conductors by Mel'nikov (1980), Anderson et al (1980) and Abrikosov (1981). Following an invariant imbedding procedure, we derive an expression for the probability 
distribution of these 'Sinai' fluctuations of resistance for $d=1$. We find that variance exponentially dominates the resistance as the length tends to infinity, while the mean conductance stays infinite for all lengths due presumably to Azbel resonance (Azbel 1983). We then extend the treatment to higher dimensions in a Migdal-Kadanoff bond moving approximation (Shapiro 1986). We find that the fluctuations dominate the mean value even at the mobility edge. It appears that one must consider all the cumulants to describe the behaviour of resistance at the mobility edge (see Al'tshuler $e$ al 1986 and references therein) necessitating a deeper analysis of the one-parameter scaling ansatz (Abrahams et al 1979). Next, we show that an external electric field tends to harness these fluctuations through its delocalizing effect. Finally, we consider the $1 / f$ noise generated by these fluctuations at the surface of a disordered semi-infinite conductor. Direct experimental probing of "Sinai" fluctuations is briefly discussed at the end.

\section{One-dimensional disordered conductors}

Consider a one-dimensional (1-d) disordered conductor of length $L$, terminated at both ends in perfect leads. We can view the whole sample as a one-channel potential scatterer characterized by an amplitude reflection coefficient $R(L)=|R(L)| \exp (i \theta(L))$. The resistance $(\rho)$ in units of $\pi \hbar / e^{2}$ can then be expressed in terms of this emergent quantity $R(L)$ as

$$
\rho(L)=\frac{|R(L)|^{2}}{1-|R(L)|^{2}}
$$

The $L$-dependence of $R(L)$ can now be studied by the method of invariant imbedding (Kumar 1985) which directly addresses the emergent quantity. The imbedding equation for $R(L)$ is

$$
\frac{\mathrm{d} R(L)}{\mathrm{d} L}=-\frac{1}{2 i k}\left[\left(k^{2}(L)-k^{2}\right)\left(1+R^{2}(L)\right)+2\left(k^{2}(L)+k^{2}\right) R(L)\right],
$$

where $\hbar^{2} k^{2} / 2 m \equiv E$ is the incident electron energy $\left(=\right.$ the Fermi energy at $T=0^{\circ} \mathrm{K}$ ) and $k(x)$ is the local wave number given by $\hbar^{2} k^{2}(x) / 2 m=E-V(x)$, and $V(x)$ is the random potential assumed gaussian white noise with $\langle V(x)\rangle=0,\left\langle V(x) V(x)^{\prime}\right\rangle=V_{0}^{2} \delta\left(x-x^{\prime}\right)$.

Equation (2) is a stochastic differential equation. As we are interested in the probability distribution $W_{\rho}(\rho)$ of $\rho(L)$, and therefore, $W_{r}(r)$ of $r(L)=|R(L)|^{2}$, we have to go over to the associated Fokker-Planck equation for $W_{r}(r)$ which is readily obtained as

$$
\frac{\partial W_{r}}{\partial l}=r(1-r)^{2} \frac{\partial^{2} W_{r}}{\partial r^{2}}+(1-r)(1-5 r) \frac{\partial W_{r}}{\partial r}+2(2 r-1) W_{r},
$$

where we have introduced the dimensionless length $l=L / \xi, \xi=\hbar^{2} E / 2 m V_{0}^{2}$, the localization length. Simple change of variable gives for $W_{\rho}$

$$
\frac{\partial W_{\rho}}{\partial l}=\rho(1+\rho) \frac{\partial^{2} W_{\rho}}{\partial \rho^{2}}+(1+2 \rho) \frac{\partial W_{\rho}}{\partial \rho},
$$


which is our central exact result. From (4), we get the moments $\rho_{n} \equiv\left\langle\rho^{n}\right\rangle$ by multiplying both sides of the equation by $\rho^{n}$ and integrating by parts on the right-hand side. We have the first two moments

$$
\begin{aligned}
& \rho_{1}=\frac{1}{2}(\exp (2 l)-1) \\
& \rho_{2}=\frac{1}{12}(\exp (6 l)-6 \exp (2 l)+4) .
\end{aligned}
$$

From (5) we see that for $l \ll 1$ the $l$-dependence is ohmic which is understandable as the cumulative effect of coherence has not built up yet. For $l \gg 1$, the mean resistance grows exponentially, and variance exponentially dominates the mean indicating nonadditivity and non-self averaging. The asymptotic form of the full probability distribution is $(l \gg 1, \rho \gg 1)$

$$
W_{\rho}(\rho) \sim \frac{\exp (-l / 4)}{(4 \pi l)^{1 / 2}} \rho^{-1 / 2} \exp \left(-\frac{1}{4 l} \ln ^{2} \rho\right)
$$

which gets broader as $l \rightarrow \infty$. Equation (6) shows that the $\log \rho$ (proportional to inverse of localization length) does obey the central limit theorem-the levelling property of the logarithm!

It is interesting to examine the effect of an external electric field $F$, which is easily included by the replacement $V(x) \rightarrow V(x)-e F x$. The modified probability distribution $W_{p}^{F}$ obeys (Vijayagovindan et al 1986)

$$
\begin{aligned}
\frac{\partial W_{\rho}^{F}}{\partial L}= & \frac{1}{\eta\left(1+\frac{|e| F L}{E}\right)}\left[\frac { \partial } { \partial \rho } \left\{\rho ( 1 + \rho ) \left(\frac{\partial W_{\rho}^{F}}{\partial \rho}\right.\right.\right. \\
& \left.\left.\left.+{\frac{|e| F \eta W_{\rho}^{F}}{E\left(1+\frac{1}{1+|e| F L / E}\right)}}^{1 / 2}\right)\right\}\right]
\end{aligned}
$$

with

$$
\eta=E^{2} / k^{2} V_{0}^{2}
$$

The interesting point to note is that the distribution $W_{\rho}^{F}$ saturates to a limiting Poissonian form in the limit $L \rightarrow \infty$. Also, the mean resistance given by

$$
\rho_{1}^{F} \simeq \frac{1}{2}\left[\left(1+\frac{|e| F L}{E}\right)^{\frac{2 E}{|e| F \xi}}-1\right]
$$

interpolates between the exponential and a power-law length dependence as the field $F$ is increased, the cross-over being at $2 E /|e| F \xi \simeq 1$. This is, of course, a manifestation of the field-induced weakening of localization (Soukoulis et al 1983). 


\section{Fluctuations at the mobility edge $(\mathrm{D}>1)$}

Our starting points is the 1-d equation (4). We assume for simplicity an anisotropic case where the disorder is only along the direction of the externally impressed current. Consider a $d$-dimensional hypercube $b^{d}$, and ignore the $b^{d-1}$ bonds transverse to the chosen direction of current flow (classically these bonds connect equipotential points and hence play no role). We now combine the $b$ units in series along the direction of current flow quantum mechanically according to (4), and then combine the $b^{d-1}$ parallel chains in parallel classically (Kumar and Jayannavar 1986; Shapiro 1986). Thus, we get the scaling relation

$$
\left(\rho_{(b)}^{(d)}\right)^{-1}=\sum_{i=1}^{b^{d-1}}\left(\rho_{i}^{(1)}(b)\right)^{-1}
$$

Making $b$ a continuous variable then gives the evolution

$$
\begin{aligned}
\frac{\partial K_{\rho}^{(d)}}{\partial \ln l}= & -(d-1) x \frac{\partial K}{\partial x}+\frac{1}{2}\left[x^{2} \frac{\partial^{2} K_{\rho}^{(d)}}{\partial x^{2}}+x^{2}\left(\frac{\partial K_{\rho}^{(d)}}{\partial x}\right)^{2}\right. \\
& \left.+x(2-x) \frac{\partial K_{\rho}^{(d)}}{\partial x}-x\right] \ln \left(1+2 \rho_{1}^{(d)}\right),
\end{aligned}
$$

where we have introduced the characteristic function

$$
K_{\rho}^{(d)}=\ln \int_{0}^{\infty} \exp (-x \rho) W_{\rho}^{(d)} \mathrm{d} \rho
$$

and $\rho_{1}^{(d)}$ in the mean resistance of the $d$-dimensional system.

It is now readily seen that (11) has a fixed point distribution obtained by setting the left hand side to zero. The associated fixed-point probability $W_{\rho}^{*(d)}$ is given by a power law

$$
W_{\rho}^{*(d)}=\frac{(1+\rho)^{-\alpha}}{(\alpha-1)}, \quad \text { with } \quad \alpha=2(d-1) /\left(1+2 \rho_{1}^{*(d)}\right)
$$

where $\rho_{1}^{*(d)}$ is the critical resistance (mobility edge). Thus, the mean is finite but all cumulants are infinite. This extreme dominance of fluctuation may be due to assumed anisotropy that presumably over-emphasizes the one-dimensional character. This may also account for the one-parameter scaling nature of solution (12). Further analysis shows that even on the metallic side, $\rho_{1}^{(d)}<\rho_{1}^{*(d)}$, the variance remains comparable with the mean.

We have attempted the general case of isotropic disorder and our results (Kumar and Jayannavar 1985) do not support the one-parameter scaling ansatz. 


\section{Sinai fluctuations and $1 / f$-noise}

Yet another manifestation of these fluctuations is the $1 / f$-noise resulting from the resonant back-scattering of positive energy electrons at the interface between a semiinfinite disordered system and a perfect lead. The point is that the positive energy Anderson localized states deep inside the bulk appear as resonant states nearer the interface. Resonant reflection leads to energy dispersive phase shift $\theta$ and therefore, to a distribution of the delay time $(\Delta t)$. The latter results in non-cancellation of instantaneous surface currents resulting from the incident and retarded reflected waves. The noise spectrum turns out to have the $1 / f$ character. We have derived the probability distribution $W(\tau)$ :

with

$$
W(\tau)=\frac{\lambda \exp (\lambda \arctan \tau)}{(\exp (\lambda \pi / 2)-1)\left(1+\tau^{2}\right)},
$$

$$
\tau=\frac{2 E(\Delta t)}{\sqrt{3} \hbar}, \quad \lambda=\frac{8}{\sqrt{3}} k \xi .
$$

The long power law tails gives infinite delay time in-the-mean which corroborates with the fact noted earlier that the mean conductance is infinite even for infinite sample length. Noise spectrum for currents then turns out to be linear in frequency, giving a $1 / f$ noise for charge or voltage fluctuations.

\section{Discussion}

The resistance fluctuation, its dominance over the mean and the consequent possible deviation from the one-parameter scaling ansat $z$ are due ultimately to the coherence effects associated with the wave nature of electrons. As such we expect acoustic and optical analogues of these effects. A direct experimental observation of 'Sinai' fluctuations is, however, possible by introducing disorder coulometrically and current cycling the sample, keeping the net ampere-hours (charge-transferred) constant, (e.g. in $\cdot \mathrm{Ag}_{2+\delta} S$ ). Thus, we naturally have macroscopically identical samples but differing in the microscopic complexions (i.e., depending on where the ions are lodged). Then, one would expect identical resistance at higher temperatures but a large scatter in the lowtemperature resistances.

Another interesting experiment would be to observe the time-translation of 'Sinai' fluctuations at non-zero temperature possible under the condition that the sample length be small compared to the inelastic mean free path (or better still the Thouless length) but the transit-time of the electron through the sample be smaller than the period of the dominant thermal phonon. Then, the lattice-potential modulation by phonons is seen only adiabatically by the electrons (no inelastic scattering) and the successive electrons see effectively different realizations of the random potential giving an excess noise. We could call this scintillation noise somewhat after the twinkling of stars. 


\section{References}

Abrahams E, Anderson P W, Licciardello D C and Ramakrishnan T V 1979 Phys. Rev. Lett. 42673 Abrikosov A A 1981 Solid State Commun. 37997

Al'tshuler B L, Kravstov V E and Lerner I V 1986 Academy of Sciences of the USSR (Leningrad Nuclear Physics Institute) Preprint No. 1229 (and references cited therein)

Anderson P W, Thouless B J, Abrahams E and Fisher D S 1980 Phys. Rev. B22 3519

Azbel M Ya 1983 Solid State Commun. 45527

Jayannavar A M, Vijayagovindan G V and Kumar N 1987 (to appear)

Kumar N 1985 Phys. Rev. B31 5513

Kumar N and Jayannavar A M 1986 J. Phys. C19 L85

Kumar N and Mello P A 1985 Phys. Rev. B31 3109

Mel'nikov V I 1980 Pis'ma Zh. Eksp. Teor. Fiz. 32244

Pendry J B, Kirkman P D and Castano E 1986 Phys. Rev. Lett. 572983

Shapiro B 1986 Phys. Rev. B34 4394

Soukoulis C M, Jose J V, Economon E N and Sheng P 1983 Phys. Rev. Lett. 59764

Vijayagovindan G V, Jayannavar A M and Kumar N 1987 Phys. Rev. B 352029 